\title{
Efeito do treino de alcance combinado com estimulação elétrica em lactentes com paralisia braquial perinatal: estudo experimental de caso único
}

\author{
Effect of reaching training combined with electrical stimulation in infants with brachial \\ plexus palsy: a single subject design \\ Efecto del entrenamiento de alcance combinado con electroestimulación en los lactantes con \\ parálisis braquial perinatal: un estudio experimental de caso único \\ Rejane Vale Gonçalves', Renata Calheiros de Araujo², Vivianne Kellen Gonçalves Ferreira ${ }^{3}$
}

RESUMO | O objetivo deste estudo foi avaliar o efeito do treino de alcance combinado à estimulação elétrica funcional (FES) na movimentação ativa do membro superior de lactentes com paralisia braquial perinatal (PBP). Trata-se de estudo experimental de caso único do tipo A-B com follow-up. Foram documentados semanalmente dados relativos à função do membro superior (Escala de Movimento Ativo) de dois lactentes, uma menina e um menino de 7 e 10 meses de idade, respectivamente. Após seis avaliações, três vezes por semana, durante duas semanas, na linha de base (A), a intervenção consistiu em aplicar FES Neurodyn ${ }^{\circledast}$ no músculo deltoide em combinação com treino de alcance de objetos, incentivando abdução e flexão anterior de ombro acima de $90^{\circ}$, durante seis semanas, totalizando 15 atendimentos (B). Os lactentes foram reavaliados duas vezes após um período de 15 e 30 dias sem intervenção (follow-up). A pontuação total obtida em cada dia de avaliação foi representada graficamente. Houve boa aceitação da eletroestimulação, sem nenhum efeito colateral. Ambos os lactentes apresentaram melhora da movimentação ativa do membro superior afetado após a intervenção e os ganhos foram mantidos no follow-up, com aumento de 9 e 7 pontos para os lactentes 1 e 2 , respectivamente. Seis semanas de intervenção resultaram em mudanças individuais na função do membro superior dos lactentes, principalmente no aumento da amplitude de movimento ativo em flexão e abdução de ombro e flexão de cotovelo. Os resultados deste estudo sugerem o uso de estimulação elétrica como coadjuvante do treino do uso do membro superior afetado de lactentes com PBP.

Descritores | Neuropatias do Plexo Braquial; Estimulação Elétrica; Fisioterapia.

ABSTRACT I This study aimed to evaluate the effect of reaching training combined with functional electrical stimulation (FES) on active upper limb movement in infants with perinatal brachial palsy (PBP). Experimental singlecase A-B design with follow-up. Two infants participated in the study, one girl of 7 months and one boy of 10 months of age. Data on infant's upper limb function (Active Movement Scale) were documented weekly. After six baseline assessments (A), 3 times per 2 weeks, intervention

'Faculdade Ciências Médicas de Minas Gerais (FCMMG) - Belo Horizonte (MG), Brasil. E-mail: rejanevalegoncalves@gmail.com. Orcid: 0000-0002-3455-5405

${ }^{2}$ Faculdade Ciências Médicas de Minas Gerais (FCMMG) - Belo Horizonte (MG), Brasil. E-mail: renatynhacalheiros@gmail.com. Orcid: 0000-0001-9512-4388

${ }^{3}$ Faculdade Ciências Médicas de Minas Gerais (FCMMG) - Belo Horizonte (MG), Brasil. E-mail: vivianne.kgf@gmail.com. Orcid: 0000-0001-6482-7400

Um resumo de parte deste trabalho foi apresentado no formato de pôster no V Congresso Brasileiro de Fisioterapia Neurofuncional que ocorreu nos dias 11, 12 e 13 de outubro de 2018 em Florianópolis, Santa Catarina. 
consisted of applying FES Neurodyn ${ }^{\circledast}$ to the deltoid muscle combined with objects reach training, encouraging abduction and anterior shoulder flexion above 90, for six weeks, totaling 15 visits (B). The infants were reevaluated twice after a period of 15 and 30 days without intervention (follow up). The total score obtained on each evaluation day was plotted graphically. Electrostimulation was well accepted, with no side effects. Both infants showed improvement in the active movement of the affected upper limb after the intervention and the gains were maintained at follow-up, an increase of 9 and 7 points for infants 1 and 2 , respectively. Six weeks of intervention resulted in individual changes in infant's upper limb function, mainly increased active range of motion in shoulder flexion and abduction and elbow flexion. The results of this study suggest the use of electrical stimulation as an adjunct to training the use of the affected upper limb of infants with PBP.

Keywords | Brachial Plexus Neuropathies; Electrical Stimulation; Physical Therapy Specialty.

RESUMEN I El objetivo de este estudio fue evaluar el efecto del entrenamiento de alcance combinado con electroestimulación funcional (FES) sobre el movimiento activo de la extremidad superior de los lactantes con parálisis braquial perinatal (PBP). Este es un estudio experimental de caso único de tipo A-B con follow-up.
Se registraron semanalmente datos sobre la función del miembro superior (escala de movimiento activo) de dos lactantes, una niña y un niño de 7 y 10 meses de edad, respectivamente. Después de seis evaluaciones, tres veces semanales, durante dos semanas, en la línea base (A), la intervención consistió en aplicar FES Neurodyn ${ }^{\circledast}$ en el músculo deltoides combinado con entrenamiento de alcance de objetos para estimular la abducción y la flexión anterior del hombro por encima de $90^{\circ}$, durante seis semanas, lo que totalizó 15 visitas (B). Se reevaluó a los lactantes dos veces tras el período de 15 y 30 días sin intervención (follow-up). El puntaje total que se obtenía en cada evaluación se representaba gráficamente. Hubo buena aceptación de la electroestimulación, sin efectos secundarios. Ambos lactantes tuvieron mejora en el movimiento activo de la extremidad superior afectada tras la intervención, y los beneficios se mantuvieron en el follow-up, con un aumento de 9 y 7 puntos para los lactantes 1 y 2 , respectivamente. Seis semanas de intervención produjeron cambios individuales en la función del miembro superior de los lactantes, principalmente el aumento de la amplitud del movimiento activo en la flexión y abducción del hombro y la flexión del codo. Los resultados sugieren usar la electroestimulación como complemento en el entrenamiento de la extremidad superior afectada de lactantes con PBP. Palabras clave | Neuropatías del Plexo Braquial; Estimulación Eléctrica; Fisioterapia.

\section{INTRODUÇÃO}

A paralisia braquial perinatal (PBP) refere-se à paralisia flácida do membro superior, secundária a uma lesão traumática em uma ou mais raízes do plexo braquial durante o período perinatal ${ }^{1}$. A incidência da $\mathrm{PBP}$ varia de 0,4 a 4,6 por 1.000 nascidos vivos ${ }^{2,3}$. O tipo mais comum de PBP é a paralisia de Erb, que afeta as raízes de $\mathrm{C} 5$ e C6 e corresponde a $50-60 \%$ dos casos ${ }^{1}$. A PBP é geralmente transitória, com recuperação parcial ou completa das funções do membro superior nos três primeiros meses de vida, caso tenha ocorrido neuropraxia ${ }^{4}$. Entretanto, em 10-30\% dos casos não há recuperação espontânea. Portanto, o lactente apresentará dificuldades no uso do membro superior afetado, fraqueza muscular e poderá desenvolver contraturas e deformidades ${ }^{5,6}$. Nesses casos, a lesão poderá ter sido do tipo axoniotmese ou neurotmese. Esta última apresenta pior prognóstico e o lactente provavelmente necessitará de intervenção cirúrgica ${ }^{4}$.

A fisioterapia tem um papel essencial na estimulação do membro superior afetado de lactentes com PBP de forma a incentivar a movimentação ativa, diminuir possíveis movimentos compensatórios e aumentar a força muscular ${ }^{7,8}$. Estudos sugerem que estratégias como descarga de peso e mudança de decúbito e posicionamento poderiam ser adotadas para melhorar a atividade muscular do lactente com $\mathrm{PBP}^{8,9}$. A estimulação elétrica é um dos recursos que podem ser utilizados para aumentar a força em membros plégicos ou paréticos, sendo muito usada em crianças com paralisia cerebral ${ }^{10}$. É possível que o uso de estimulação elétrica funcional (FES) possa otimizar também o processo de reabilitação de crianças com $\mathrm{PBP}$, pois estimula a contração sustentada do músculo e recruta as fibras do tipo II (i.e., de contração rápida) ${ }^{11}$.

A literatura apresenta estudos sobre estimulação elétrica em adultos com lesão traumática de plexo braquial ${ }^{12}$, sendo escassos os estudos em crianças, principalmente em lactentes. $\mathrm{O}$ único estudo encontrado pelos autoras sobre o uso de FES em lactentes antes do segundo ano de vida foi o de Berggren e Baker ${ }^{13}$. Eles relataram o caso de um lactente com PBP total que foi acompanhado durante os dois primeiros anos de vida. Quando tinha 
entre 11 e 14 meses de idade o lactente foi submetido à FES em flexores e extensores de cotovelo e punho, tendo como desfecho a melhora na movimentação ativa dessas articulações ${ }^{13}$. Estudos sugerem que, quanto mais jovem é a criança, maior é a capacidade de recuperação da função do membro superior afetado pela $\mathrm{PBP}^{14}$. Entretanto, não está claro como o tratamento conservador pode auxiliar nesse processo de recuperação, pois não foram encontrados estudos que investigaram o efeito do treino do uso do membro superior afetado em lactentes com esse tipo de lesão ${ }^{15}$. O objetivo deste estudo foi investigar o efeito da FES combinada com o treino de alcance na movimentação ativa do membro superior afetado de lactentes com PBP.

\section{METODOLOGIA}

\section{Delineamento do estudo}

Foi realizado um estudo experimental de caso único do tipo $\mathrm{AB}$ com follow-up, sendo a fase $\mathrm{A}$ referente ao baseline (duas semanas, avaliações três vezes por semana), a fase B referente à intervenção (seis semanas, avaliações e intervenção três vezes por semana) e o follow-up referente a duas reavaliações, 15 e 30 dias após o término da intervenção (Quadro 1). Os responsáveis pelos lactentes assinaram o Termo de Consentimento Livre e Esclarecido (TCLE) antes da participação no estudo.

Quadro 1. Delineamento do estudo: distribuição das avaliações e dias de intervenção ao longo das semanas

\begin{tabular}{|c|c|c|c|c|c|c|c|c|c|c|c|c|c|c|c|c|c|c|c|c|c|c|c|c|c|c|c|c|}
\hline & \multicolumn{6}{|c|}{ Fase A: Baseline } & \multicolumn{18}{|c|}{ Fase B: Intervenção } & \multicolumn{4}{|c|}{ Follow-up } \\
\hline Semana & \multicolumn{3}{|c|}{1} & \multicolumn{3}{|c|}{2} & \multicolumn{3}{|c|}{3} & \multicolumn{3}{|c|}{4} & \multicolumn{3}{|c|}{5} & \multicolumn{3}{|c|}{6} & \multicolumn{3}{|c|}{7} & \multicolumn{3}{|c|}{8} & 9 & 10 & 11 & 12 \\
\hline Avaliação & $x$ & $x$ & $x$ & $x$ & $x$ & $x$ & $x$ & $x$ & $x$ & $x$ & $x$ & $x$ & $x$ & $x$ & $x$ & $x$ & $x$ & $x$ & $x$ & $x$ & $x$ & $x$ & $x$ & $x$ & & $x$ & & $x$ \\
\hline Intervenção & & & & & & & X & $x$ & $x$ & $x$ & $x$ & $x$ & $x$ & $x$ & $x$ & X & $x$ & $x$ & $x$ & $x$ & $x$ & $x$ & X & $x$ & & & & \\
\hline
\end{tabular}

\section{Amostra}

Os lactentes foram selecionados dentro da clínicaescola de fisioterapia da Faculdade Ciências Médicas de Minas Gerais (FCMMG). Foram incluídos no estudo lactentes com idade entre 4 e 12 meses, com PBP do tipo Erb (C5 e C6), grupo 1, de acordo com a classificação de Narakas ${ }^{16}$ e lesão do tipo axoniotmese, conforme diagnóstico dado pelo neurologista. Os lactentes incluídos já estavam inseridos em um programa de reabilitação quando iniciaram a participação no estudo.

\section{Procedimentos}

Inicialmente foi realizada uma entrevista com a família para coletar dados referentes à idade cronológica, idade gestacional, peso, estatura, perímetro cefálico e Apgar de cada lactente. A amplitude de movimento passivo dos principais músculos do membro superior afetado foi avaliada para documentar possíveis encurtamentos musculares. Além disso, foi explicado ao responsável pelo lactente que o uso da corrente elétrica poderia provocar uma sensação de formigamento e que durante a intervenção as expressões faciais do lactente e sua interação com os terapeutas e os objetos seria monitorada o tempo todo, de forma a identificar possível desconforto, mesmo que o lactente não o demonstrasse por meio do choro ou de compensações.
Os lactentes foram submetidos a três avaliações semanais da movimentação ativa do membro superior afetado durante as fases $\mathrm{A} \mathrm{e} \mathrm{B} \mathrm{e} \mathrm{a} \mathrm{duas} \mathrm{avaliações} \mathrm{correspondentes} \mathrm{ao} \mathrm{follow-}$ up. Todas as avaliações foram filmadas para que, por meio de análise de vídeo, fosse aplicada a escala de movimento ativo (EMA $)^{17}$. A EMA é um instrumento que avalia a movimentação ativa do membro superior afetado do lactente e que pode ser utilizada em crianças desde o nascimento até a adolescência. A pontuação de 15 movimentos articulares do membro superior é graduada em uma escala que varia de zero a sete, totalizando 105 pontos, sendo baseada na quantidade de movimentação observada dentro da amplitude de movimento (ADM) passiva disponível. A movimentação ativa realizada com a gravidade minimizada é pontuada de zero a quatro, enquanto movimentos que vencem a ação da gravidade são pontuados de cinco a sete ${ }^{17}$. A EMA foi utilizada para documentar o principal desfecho de interesse deste estudo, que está incluído no domínio de estrutura e função corporal da Classificação Internacional de Funcionalidade, Incapacidade e Saúde (CIF) ${ }^{18}$. Trata-se de uma escala válida e confiável ${ }^{17}$, capaz de documentar a evolução clínica do lactente durante o primeiro ano de vida.

A avaliação incluiu também a análise visual da qualidade dos movimentos realizados com o membro acometido de forma a observar se o lactente apresentava movimentos compensatórios, tais como elevação de ombro, abdução escapular excessiva e se conseguia manter o cotovelo próximo ao corpo, além das posturas e transferências que os lactentes conseguiam realizar de acordo com sua idade. Essas 
informações foram utilizadas para conduzir a intervenção de forma a favorecer a movimentação ativa ou ativo-assistida do membro superior afetado, de acordo com o esperado para a idade do lactente. As avaliações foram realizadas por uma fisioterapeuta com mais de 10 anos de experiência na avaliação de lactentes com PBP. Por sua vez, a intervenção foi realizada pelos acadêmicos que já tinham familiaridade com o lactente e eram responsáveis pelo atendimento no período do estágio, sob supervisão direta de um professor.

A intervenção consistiu no uso de FES combinado com incentivo à movimentação ativa do lactente em atendimentos de 50 minutos por dia, três vezes por semana, durante seis semanas. $\mathrm{O}$ equipamento utilizado foi o FES Neurodyn ${ }^{\circledR}$ digital de seis canais. Dois eletrodos esféricos autoadesivos de $3 \mathrm{~cm}$ de diâmetro foram fixados: o primeiro no músculo deltoide médio, logo abaixo do acrômio, e o segundo em posição inferior ao primeiro, a uma distância de aproximadamente o tamanho do eletrodo. Inicialmente a frequência foi ajustada para 10 pulsos por segundo (pps) e a intensidade foi aumentada até provocar contração muscular visível, de forma a se localizar o melhor posicionamento dos eletrodos. Em seguida, a intensidade foi diminuída e a frequência de estímulo foi ajustada para 30pps para atingir uma contração muscular tetânica suave em abdução do ombro. A corrente elétrica utilizada foi a simétrica, com duração do pulso de 300 microssegundos. A intensidade (amplitude da corrente em 1.000 amperes) foi ajustada de forma individualizada conforme a tolerância de cada lactente, variando entre 15 e 30 mil amperes. $\mathrm{O}$ tempo $\mathrm{ON}$ foi de cinco segundos, com rampa de subida de dois segundos e rampa de descida de dois segundos; o tempo OFF foi de 10 segundos, durante 20 minutos. Sempre que a corrente estava passando o lactente era incentivado a alcançar algum objeto $^{19}$. A Figura 1 ilustra o posicionamento dos eletrodos e a intervenção da FES combinada com o incentivo ao uso do membro superior afetado (Figura 1).

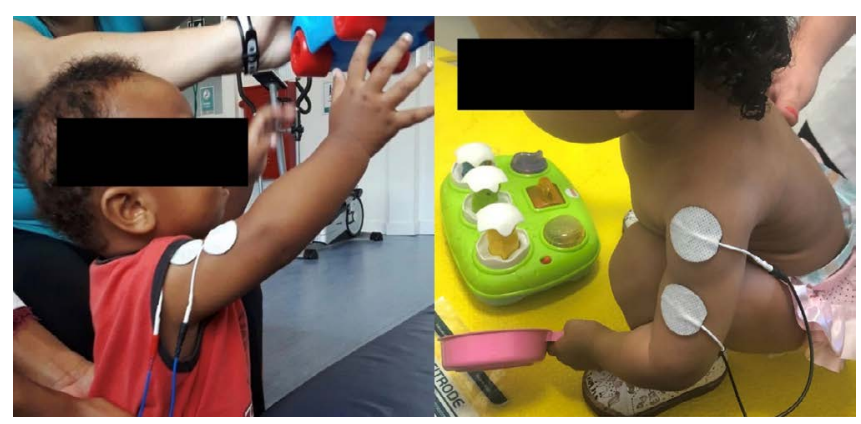

Figura 1. Posicionamento dos eletrodos autoadesivos: o primeiro fixado no músculo deltoide logo abaixo do acrômio e o segundo a uma distância de aproximadamente de $3 \mathrm{~cm}$ do primeiro eletrodo

$\mathrm{O}$ atendimento do lactente tinha duração de 50 minutos, sendo 20 minutos de treino de alcance combinado com FES e mais 30 minutos de treino de alcance sem eletroterapia, pois o uso da FES por mais de 20 minutos consecutivos poderia provocar fadiga muscular nos lactentes ${ }^{20}$. Os detalhes da intervenção são descritos no Quadro 2.

Quadro 2. Descrição da intervenção

\begin{tabular}{|l|l|l|l|l}
\hline \multicolumn{1}{c|}{ Intervenção } & Duração & \multicolumn{1}{c|}{$\begin{array}{c}\text { Objetos a serem } \\
\text { alcançados }\end{array}$} & $\begin{array}{c}\text { Posição na qual os } \\
\text { objetos eram colocados }\end{array}$ & $\begin{array}{l}\text { Posturas nas quais o lactente era } \\
\text { estimulado a alcançar os objetos }\end{array}$ \\
\hline $\begin{array}{l}\text { Treino de alcance } \\
\text { combinado com FES }\end{array}$ & 20 minutos & $\begin{array}{l}\text { Sentado } \\
\text { Quatro apoios } \\
\text { Argolas, carrinhos, bolas } \\
\text { pequenas, peças de blocos } \\
\text { de montar ou encaixar }\end{array}$ & $\begin{array}{l}\text { Acima da altura da cabeça do } \\
\text { lactente de forma a incentivar } \\
\text { que ele pegasse o objeto } \\
\text { realizando flexão anterior ou } \\
\text { abdução de ombro acima de } 90^{\circ}\end{array}$ & $\begin{array}{l}\text { Durante transferências como } \\
\text { agachar e levantar, passar de } \\
\text { deitado para sentado e passar de } \\
\text { sentado para quatro apoios }\end{array}$ \\
\hline Treino de alcance sem FES & 30 minutos & &
\end{tabular}

\section{Análise estatística}

A amostra foi descrita em termos de idade, sexo, idade gestacional, peso, estatura, perímetro cefálico e Apgar. A pontuação total obtida por cada lactente na EMA em cada dia de avaliação foi representada graficamente. Uma inclinação (celeration line) foi calculada para a fase de baseline para determinar uma tendência desenvolvimental e sua trajetória foi estendida para as outras duas fases. A diferença entre a fase de baseline e de intervenção foi determinada pela análise visual da proporção de pontos na fase de intervenção que se situavam acima ou abaixo da linha de tendência.

\section{RESULTADOS}

Participaram deste estudo dois lactentes, sendo o lactente 1 do sexo feminino, com sete meses de idade, e o lactente 2 do sexo masculino, com 10 meses de idade. O lactente 1 nasceu de parto vaginal, com idade gestacional de 40 semanas, peso ao nascimento de $3.650 \mathrm{~g}$, estatura 
de $51 \mathrm{~cm}$, perímetro cefálico de $36 \mathrm{~cm}$ e Apgar de 9 no primeiro e no quinto minuto de vida. $\mathrm{Na}$ avaliação inicial foi documentado encurtamento muscular de trapézio superior, bíceps braquial, redondo maior, peitoral maior e pronadores de antebraço esquerdo. $\mathrm{O}$ membro superior afetado era hipomóvel e mantido em rotação interna e elevação do ombro, semiflexão do cotovelo e pronação do antebraço. O lactente apresentava limitação para realizar descarga de peso durante as transferências e dificuldade para alcance, pois isso era realizado apenas quando o objeto era colocado próximo ao seu corpo. Quando o alcance exigia movimento de flexão anterior de ombro o lactente não conseguia realizá-lo. A manipulação de objetos com membro afetado era difícil devido à incapacidade do lactente de realizar supinação de antebraço, resultando em negligência.

O lactente 2 nasceu de parto vaginal, com idade gestacional de 40 semanas, peso ao nascimento de $4.775 \mathrm{~g}$, estatura de $53 \mathrm{~cm}$, perímetro cefálico de $38 \mathrm{~cm}$ e Apgar de 8 no primeiro minuto e 9 no quinto minuto. $\mathrm{Na}$ avaliação inicial foi documentado encurtamento muscular de peitoral maior e redondo maior, cotovelo em semiflexão e hiperatividade de trapézio superior. O lactente conseguia alcançar objetos até a metade da amplitude de movimento de flexão anterior e abdução de ombro contra a força da gravidade, e não conseguia realizar supinação de antebraço de forma ativa.

Os dois lactentes que participaram do estudo toleraram bem a estimulação elétrica e não apresentaram nenhum efeito colateral, expressão de incômodo ou dor. Ambos foram submetidos a 15 sessões de intervenção durante o período de seis semanas, pois houve três faltas ao longo desse período devido a dificuldades pessoais dos responsáveis. Após a intervenção foi documentada melhora da movimentação ativa do membro superior afetado nos dois lactentes, avaliada por meio da EMA, principalmente aumento da amplitude de movimento ativo em flexão e abdução de ombro e flexão de cotovelo. Essa melhora foi mantida nas duas reavaliações realizadas 15 e 30 dias após o término da intervenção. Os ganhos foram diferentes para cada lactente, pois a capacidade de realizar movimento ativo com o membro superior afetado era distinta entre eles. O lactente 1 conseguiu alcançar objetos realizando flexão anterior de ombro contra a força da gravidade em menos da metade da amplitude e apresentou maior uso do membro superior afetado em comparação ao início do estudo. O lactente 2 , que já conseguia realizar alcance até a metade da amplitude de movimento de flexão anterior e abdução de ombro no início do estudo, foi capaz de realizar o alcance até o final da amplitude de movimento após a intervenção. Os dois grupos musculares que menos apresentaram mudanças após a intervenção foram os rotadores externos de ombro e supinadores de antebraço, pois os lactentes apresentaram esses movimentos apenas com a ação da gravidade minimizada. A Figura 2 apresenta os gráficos da pontuação total obtida na EMA dos participantes 1 e 2 ao longo de todo o período do estudo.
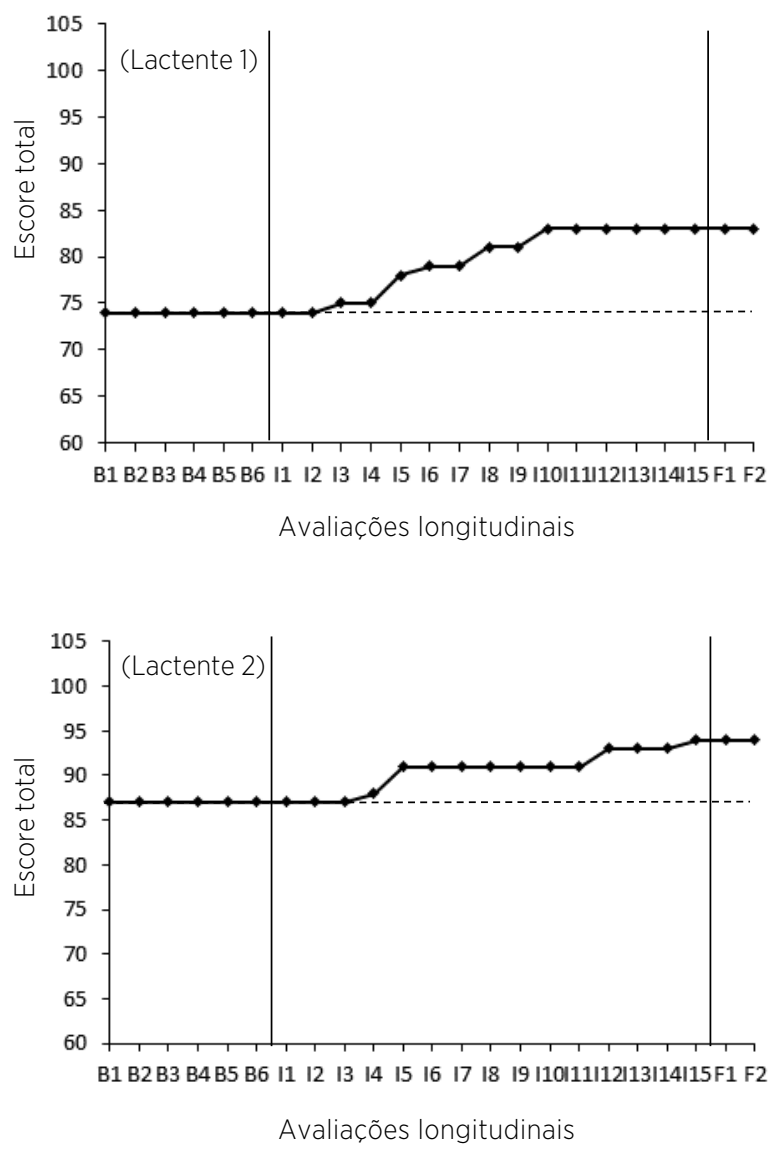

Figura 2. Gráficos do escore total da escala de movimento ativo (EMA) dos lactentes 1 e 2 durante as 23 avaliações longitudinais. Os escores obtidos no baseline (B1-B6), intervenção (11-115) e follow-up (Fle F2) são mostrados juntos a uma linha (--.--), indicando a pontuação obtida na fase de baseline, que foi estendida para as outras duas fases. Essa linha de tendência é a celeration line

Ao final da intervenção de treino de alcance combinado com o FES, ambos os lactentes eram capazes de se manter em quatro apoios com os braços estendidos, porém o lactente 1 ainda necessitava de apoio no tronco para manter a estabilidade e realizar alcance nesta postura. Também foi observada melhora na estabilidade da cintura escapular de ambos os lactentes, de forma que o lactente 2 já conseguia realizar descarga de peso com o membro afetado e alcançar com o outro membro superior. 


\section{DISCUSSÃO}

Este estudo experimental de caso único documentou o efeito do treino de alcance combinado com a FES em lactentes com PBP. Os resultados mostraram que houve uma boa tolerância dos lactentes à estimulação elétrica, apontando que essa intervenção pode ser um recurso para potencializar o uso do membro superior afetado pela PBP. Como esperado, durante o período de intervenção os lactentes conseguiram alcançar em uma amplitude maior do que conseguiam previamente à intervenção combinada com a FES. A menor mudança foi documentada nos movimentos de rotação externa de ombro e supinação de antebraço, resultado apoiado pela literatura, que aponta que esses são os últimos grupos musculares a apresentarem recuperação da movimentação ativa em crianças com $\mathrm{PBP}^{6}$.

Este estudo propôs uma intervenção conservadora para lactentes com PBP. A literatura aponta diversos estudos sobre intervenção cirúrgica para os lactentes com PBP que não recuperaram a função do membro superior afetado após o terceiro ou sexto mês de vida ${ }^{8,21}$. Este estudo acompanhou dois lactentes que já tinham passado da idade que a literatura aponta como o período ideal para recuperação da função do membro superior no caso de lesão das raízes de $\mathrm{C} 5$ e C6, o que reforça a efetividade da intervenção proposta ${ }^{8,15}$. Entretanto, a comparação destes resultados com outros estudos que propuseram tratamento conservador no primeiro ano de vida é limitada, pois as pesquisas são escassas. Um ensaio clínico aleatorizado investigou o efeito da dosagem de um programa de exercícios que incluía movimentação passiva e ativa das articulações do membro superior afetado de lactentes com PBP. Embora não tenha havido diferença entre a realização de exercícios uma ou três vezes ao dia, os lactentes de ambos os grupos melhoraram a amplitude de movimento passiva e ativa nas reavaliações aos 3, 6 e 12 meses de idade ${ }^{22}$. Outro ensaio clínico aleatorizado com crianças maiores, entre três e cinco anos de idade, mostrou que uma intervenção que incluía exercícios de resistência e descarga de peso no membro superior afetado pela PBP não foi diferente da mesma intervenção combinada com $\mathrm{FES}^{19}$. Entretanto, a capacidade de recuperação em lactentes é superior em crianças maiores devido ao processo de recuperação neuronal que ocorre nos primeiros meses após a lesão pela $\mathrm{PBP}^{3}$.

A reabilitação de lactentes com PBP é muito distinta daquela em crianças maiores, pois os lactentes não atendem aos comandos verbais dados pelo terapeuta. Para que o lactente alcance os objetos, a intervenção precisa ser realizada em um contexto lúdico, com diferentes brinquedos, de forma que ele tenha interesse em alcançá-los. Neste estudo, era possível identificar, pela expressão facial dos lactentes, que eles estavam sentindo algo em seu membro superior enquanto a corrente elétrica estava no tempo $\mathrm{ON}$, pois o lactente olhava para a mão. A estimulação elétrica chamava a atenção do lactente para o membro superior afetado e isso favorecia o uso desse membro durante o alcance. Além disso, os lactentes não choraram ou expressaram dificuldades durante a estimulação elétrica. Portanto, uma grande contribuição deste estudo é mostrar a viabilidade do uso da FES em lactentes.

A literatura aponta que não é indicado o uso de FES por muitos minutos consecutivos devido ao risco de provocar fadiga muscular nos lactentes ${ }^{20}$. Portanto, neste estudo, após o treino de alcance com FES, o lactente era incentivado a continuar o treino sem o uso da FES. $\mathrm{O}$ atendimento fisioterapêutico com lactentes é muito dinâmico. Para mantê-los envolvidos no desempenho das atividades de alcance era necessário usar diferentes objetos a serem alcançados de acordo com seu interesse. Ademais, o lactente ativamente mudava de postura, por exemplo, transferindo-se de sentado para quatro apoios, e o terapeuta continuava o treino de alcance enquanto interagia com ele. Dessa forma, a melhora na movimentação ativa que os lactentes apresentaram pode ser atribuída ao conjunto de atividades desempenhadas que tinha como principal enfoque o treinamento de alcance, que foi realizado tanto combinado quanto sem o auxílio da estimulação elétrica.

Este estudo apresenta algumas limitações. O período da baseline de duas semanas usado pode não ter sido suficiente para estabelecer uma fase pré-intervenção estável para as variáveis estudadas. Entretanto, não foi possível usar o período de baseline igual ao período de intervenção, pois haveria risco de atrito experimental, ou seja, de ameaça à validade interna do estudo devido à maturação dos lactentes. O período de follow-up também foi curto, o que impossibilita afirmar se os ganhos obtidos foram mantidos após a última avaliação dos desfechos. Outra limitação é a amostragem pequena, que limita a generalização dos resultados. Porém, o delineamento experimental de caso único deste estudo permitiu documentar as mudanças na movimentação ativa de cada lactente, sob condições controladas, considerando suas especificidades individuais. Esse desenho de estudo é útil em condições de saúde como a PBP, pois sua prevalência após os três meses de idade é baixa devido à recuperação espontânea que ocorre na maioria dos casos. 


\section{CONCLUSÃO}

$\mathrm{O}$ treino de alcance combinado com a FES aumentou a movimentação ativa do membro superior afetado de lactentes com PBP. Houve boa tolerância à corrente elétrica e não ocorreram intercorrências durante seu uso. Seis semanas de intervenção resultaram em mudanças individuais na função do membro superior dos lactentes. Os resultados deste estudo sugerem a utilização de estimulação elétrica como coadjuvante do treino do uso do membro superior afetado de lactentes com PBP. Novos estudos devem ser realizados para confirmação dos resultados obtidos e para elucidar os benefícios da eletroterapia em lactentes com PBP.

\section{REFERÊNCIAS}

1. Abid A. Brachial plexus birth palsy: management during the first year of life. Orthop Traumatol Surg Res. 2016;102(1):S12532. doi: 10.1016/j.otsr.2015.05.008

2. Ghizoni MF, Bertelli JA, Feuerschuette OHM, Silva RM. Paralisia obstétrica de plexo braquial: revisão da literatura. ACM Arq Catarinenses Med. 2010;39(4):95-101.

3. Lagerkvist AL, Johansson U, Johansson A, Bager B, Uvebrant P. Obstetric brachial plexus palsy: A prospective, populationbased study of incidence, recovery, and residual impairment at 18 months of age. Dev Med Child Neurol. 2010;52(6):529-34. doi: 10.1111/j.1469-8749.2009.03479.x

4. Pondaag W, Malessy MJA, Van Dijk JG, Thomeer RTWM. Natural history of obstetric brachial plexus palsy: a systematic review. Dev Med Child Neurol. 2004;46(2):138-44. doi: 10.1017/ S0012162204000258

5. Coroneos CJ, Voineskos SH, Christakis MK, Thoma A, Bain JR, Brouwers MC. Obstetrical brachial plexus injury (OBPI): Canada's national clinical practice guideline. BMJ Open. 2017;7(1):e014141. doi: 10.1136/bmjopen-2016-014141

6. Hoeksma AF, Ter Steeg AM, Nelissen RGHH, Van Ouwerkerk WJR, Lankhorst GJ, De Jong BA. Neurological recovery in obstetric brachial plexus injuries: an historical cohort study. Dev Med Child Neurol. 2004;46(2):76-83. doi: 10.1017/ S0012162204000179

7. Abzug JM, Kozin SH. Evaluation and management of brachial plexus birth palsy. Orthop Clin North Am. 2014;45(2):225-32. doi: 10.1016/j.ocl.2013.12.004

8. Yang LJS. Neonatal brachial plexus palsy-management and prognostic factors. Semin Perinatol. 2014;38(4):222-34. doi: 10.1053/j.semperi.2014.04.009
9. Ibrahim Al, Hawamdeh ZM, AISharif AA. Evaluation of bone mineral density in children with perinatal brachial plexus palsy: effectiveness of weight bearing and traditional exercises. Bone. 2011;49(3):499-505. doi: 10.1016/j.bone.2011.05.015

10. Wright PA, Durham S, Ewins DJ, Swain ID. Neuromuscular electrical stimulation for children with cerebral palsy: a review. Arch Dis Child. 2012;97(4):364-71. doi: 10.1136/ archdischild-2011-300437

11. Reed B. The physiology of neuromuscular electrical stimulation. Pediatr Phys Ther. 1997;9:96-102.

12. Limthongthang R, Muennoi P, Phoojaroenchanachai R, Vathana T, Wongtrakul S, Songcharoen P. Effectiveness and safety of home-based muscle electrical stimulator in brachial plexus injury patients. J Med Assoc Thai. 2014;97:S56-61.

13. Berggren J, Baker LL. Therapeutic application of electrical stimulation and constraint induced movement therapy in perinatal brachial plexus injury: a case report. J Hand Ther. 2015;28(2):217-21. doi: 10.1016/j.jht.2014.12.006

14. Frade F, Gómez-Salgado J, Jacobsohn L, Florindo-Silva F. Rehabilitation of neonatal brachial plexus palsy: integrative literature review. J Clin Med. 2019;8(7):980. doi: 10.3390/ jcm8070980

15. Smith BW, Daunter AK, Yang LJS, Wilson TJ. An update on the management of neonatal brachial plexus palsy: replacing old paradigms: a review. JAMA Pediatr. 2018;172(6):585-91. doi: 10.1001/jamapediatrics.2018.0124

16. Al-Qattan MM, El-Sayed AA, Al-Zahrani AY, Al-Mutairi SA, Al-Harbi MS, Al-Mutairi AM, et al. Narakas classification of obstetric brachial plexus palsy revisited. J Hand Surg Eur Vol. 2009;34(6):788-91. doi: 10.1177/1753193409348185

17. Curtis C, Stephens D, Clarke HM, Andrews D. The active movement scale: an evaluative tool for infants with obstetrical brachial plexus palsy. J Hand Surg Am. 2002;27(3):470-8. doi: 10.1053/jhsu.2002.32965

18. Duff SV, DeMatteo C. Clinical assessment of the infant and child following perinatal brachial plexus injury. J Hand Ther. 2015;28(2):126-34. doi: 10.1016/j.jht.2015.01.001

19. Elnaggar RK. Shoulder function and bone mineralization in children with obstetric brachial plexus injury after neuromuscular electrical stimulation during weight-bearing exercises. Am J Phys Med Rehabil. 2016;95(4):239-47. doi: 10.1097/ PHM.0000000000000449

20. Carmick J. Clinical use of neuromuscular electrical stimulation for children with cerebral palsy, part 2: upper extremity. Phys Ther. 1993;73(8):514-22. doi: 10.1093/ptj/73.8.514

21. Pondaag W, Malessy MJA. The evidence for nerve repair in obstetric brachial plexus palsy revisited. Biomed Res Int. 2014;2014(3):1-11. doi: 10.1155/2014/434619

22. Sahin N, Karahan AY. Effect of exercise doses on functional recovery in neonatal brachial plexus palsy: A randomized controlled study. North Clin Istanb. 2018;6(1):1-6. doi: 10.14744/ nci.2017.29200 